\title{
Properties of small rRNA methyltransferase RsmD: Mutational and kinetic study
}

\author{
OLGA V. SERGEEVA, ${ }^{1}$ IRINA V. PROKHOROVA, ${ }^{1}$ YERDOS ORDABAEV, ${ }^{1}$ PHILIPP O. TSVETKOV, ${ }^{2}$ \\ PETR V. SERGIEV ${ }^{1,3}{ }^{2}$ ALEXEY A. BOGDANOV, ${ }^{1}$ ALEXANDER A. MAKAROV, ${ }^{2}$ and OLGA A. DONTSOVA ${ }^{1}$ \\ ${ }^{1}$ Lomonosov Moscow State University, Department of Chemistry and A.N. Belozersky Institute of Physico-Chemical Biology, \\ Moscow 119992, Russia \\ ${ }^{2}$ Engelhardt Institute of Molecular Biology, Russian Academy of Sciences, Moscow 119991, Russia
}

\begin{abstract}
Ribosomal RNA modification is accomplished by a variety of enzymes acting on all stages of ribosome assembly. Among rRNA methyltransferases of Escherichia coli, RsmD deserves special attention. Despite its minimalistic domain architecture, it is able to recognize a single target nucleotide G966 of the $16 \mathrm{~S}$ rRNA. RsmD acts late in the assembly process and is able to modify a completely assembled $30 S$ subunit. Here, we show that it possesses superior binding properties toward the unmodified $30 S$ subunit but is unable to bind a $30 \mathrm{~S}$ subunit modified at G966. RsmD is unusual in its ability to withstand multiple amino acid substitutions of the active site. Such efficiency of RsmD may be useful to complete the modification of a $30 \mathrm{~S}$ subunit ahead of the $30 \mathrm{~S}$ subunit's involvement in translation.
\end{abstract}

Keywords: rRNA; modification; methylation; RsmD; N2-methyl guanosine

\section{INTRODUCTION}

Ribosomal RNA is modified in all living organisms. Modified nucleotides are clustered in the most functionally important centers of the ribosome (Urlaub et al. 1997). Complexity of an organism correlates with the number of modified residues in rRNA (Decatur and Fournier 2002). The majority of modified nucleotides in Escherichia coli rRNA are various types of base and ribose methylated residues (Sergiev et al. 2011a). Methyltransferases modify rRNA at certain stages of the assembly. Some of such enzymes could act on completely deproteinized rRNA in vitro $(\mathrm{Gu}$ et al. 1999; Tscherne et al. 1999; Lesnyak et al. 2006; Sergiev et al. 2006), while others could modify assembled subunits (Weitzmann et al. 1991; Lesnyak et al. 2007). There are some documented examples of modification enzymes that have preferences for assembly intermediates (Wrzesinski et al. 1995; Desai and Rife 2006; Connolly et al. 2008; Sergiev et al. 2008; Xu et al. 2008) and, among them, one protein that prefers associated 70S ribosomes (Ero et al. 2008). It is amazing how rRNA modification machinery is

\footnotetext{
${ }^{3}$ Corresponding author.

E-mail petya@genebee.msu.su.

Article published online ahead of print. Article and publication date are at http://www.rnajournal.org/cgi/doi/10.1261/rna.032763.112.
}

built into the general pathway of ribosomal subunits' assembly and maturation. For the methyltransferases that should act at early assembly stages, it seems that the main problem is to complete modification before ribosomal proteins will bind. For modification enzymes that act on the late assembly intermediates or even on the assembled subunits, the principal problem might be to complete modification before the involvement of incompletely mature subunits in translation.

Our study is devoted to a very unusual enzyme-rRNA methyltransferase RsmD. The target of this enzyme, nucleotide G966 of the 16S rRNA, is located in a deep cleft of the $30 \mathrm{~S}$ subunit, which is occupied by a P-site bound tRNA anticodon (Weitzmann et al. 1991; Lesnyak et al. 2007). The RsmD enzyme could methylate 16S rRNA only after binding S7 and S19 proteins to rRNA (Weitzmann et al. 1991), and modification proceeds up to a completely assembled 30S subunit (Lesnyak et al. 2007). In contrast to the majority of rRNA methyltransferases, which have separate target recognition and catalytic domains (Sergiev et al. 2007), RsmD is a minimalistic enzyme which has only a small $\beta$-hairpin extension (Kumar et al. 2011) in addition to the domain responsible for the catalysis. Nevertheless, it recognizes a single nucleotide among the $4500 \mathrm{nt}$ of rRNA.

A knockout of the rsmD gene is viable, which is indicative of functional activity of the $30 \mathrm{~S}$ subunits devoid of G966 
modification (Lesnyak et al. 2007). However, 30S subunits lacking G966 modification are less effective in translation initiation and could not support some of the gene expression control mechanisms (D Burakovsky, I Prokhorova, O Sergeeva, P Sergiev, P Milon, A Bogdanov, O Dontsova, and M Rodnina, in prep.). Since $30 \mathrm{~S}$ subunits unmodified at G966 could enter into translation, it is interesting how RsmD could ensure complete modification of all nascent ribosomes before the start of translating mRNAs. Since RsmD occupies nearly the same place on the $30 \mathrm{~S}$ subunits as the P-site bound tRNA, it should also be important for methyltransferase not to affect the functioning of modified 30 S subunits. In this work, we have demonstrated that RsmD tightly binds unmodified $30 \mathrm{~S}$ subunits but not those after modification. The active center of RsmD could tolerate mutations of the conserved amino acids.

\section{RESULTS}

\section{RsmD forms a stable complex with an unmethylated $30 S$ subunit}

Recombinant protein $\mathrm{RsmD}$, containing a $\mathrm{His}_{6}$ tag on its $\mathrm{N}$ terminus, was prepared from the strain carrying the pCA24yhhF plasmid (Kitagawa et al. 2005). The resulting protein was purified according to SDS PAGE and was used in all of the following experiments. For substrate preparation, the strain lacking the $r s m D$ gene on the chromosome (Baba et al. 2006) was used, while the isogenic wild-type strain was used to prepare the control sample. It was previously shown that assembled $30 \mathrm{~S}$ subunits are a suitable substrate for pure RsmD (Lesnyak et al. 2007). To test RsmD.30S complex formation, a twofold excess of RsmD was mixed with either $30 \mathrm{~S}$ subunits or $70 \mathrm{~S}$ ribosomes in the presence of the unreactive S-adenosyl-L-methionine (SAM) analog, sinefungin. After incubation at $37^{\circ} \mathrm{C}$, the complex was subjected to sucrose density ultracentrifugation (Fig. 1A). Gradient fractions were collected, and the amount of RsmD protein was estimated by immunoblotting (Fig. 1B). The amount of ribosomal protein S4 in the fractions was estimated for comparison (Fig. 1C). A nearly 1:1 stoichiometry of the RsmD and $30 \mathrm{~S}$ subunit complex could be estimated. No complex formation with the associated $70 \mathrm{~S}$ ribosomes could be detected (Fig. 1D).

To determine $K_{d}$ values, 30 S subunits were titrated with various concentrations of RsmD protein in the presence of sinefungin and S-adenosyl-L-homocystein (SAH) (Fig. 2). An almost similar binding of RsmD could be observed for 30 S subunits unmethylated at G966 in the presence of SAH $\left(\mathrm{K}_{\mathrm{d}}=41 \pm 15 \mathrm{nM}\right)$ and sinefungin $\left(\mathrm{K}_{\mathrm{d}}=29 \pm 5 \mathrm{nM}\right)$. Small ribosomal subunits, methylated at G966, failed to bind RsmD protein with either cofactor.

To reveal $16 \mathrm{~S}$ nucleotides that are in contact with the RsmD protein, we applied the chemical and enzymatic footprinting method. 30S ribosomes devoid of G966 modification

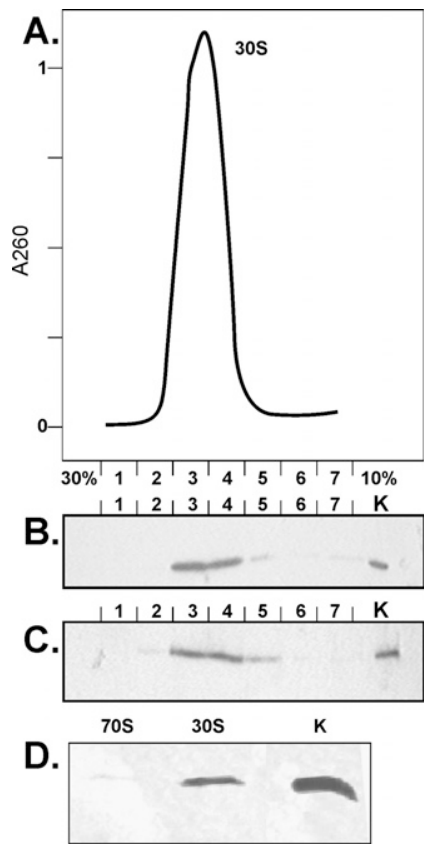

FIGURE 1. Complex formation of RsmD with 30 S subunits devoid of G966 methylation. (A) Fractionation of 30S.RsmD complex by sucrose density gradient ultracentrifugation. Shown is the optical density at $260 \mathrm{~nm}$ vs. fraction number starting from $30 \%$ to $10 \%$ sucrose. (B) Immunodetection of RsmD in the same fractions of sucrose density gradient. Lane $K$ contains 1 pmol pure RsmD. $(C)$ Immunodetection of ribosomal protein $S 4$ in the same fractions of sucrose density gradient. Lane $K$ contains 1 pmol total 30 S proteins. (D) Efficiency of RsmD complex formation with $30 \mathrm{~S}$ subunits and $70 \mathrm{~S}$ ribosomes devoid of G966 methylation. Efficiency of complex formation was monitored by immunoblotting of the corresponding sucrose density centrifugation fractions.

and its complex with RsmD and sinefungin were subjected to treatment with DMS, kethoxal, and RNases T1 and T2 (Fig. 3). A short region of the $16 \mathrm{~S}$ rRNA, proximal to the modification site, was found to be protected by RsmD in the complex.

The model of RsmD interaction with the $30 \mathrm{~S}$ subunit suggested in our publication (Lesnyak et al. 2007) seems valid. In that model, nucleotides surrounding G966 fit into the small cavity on the RsmD surface near the SAM binding site. Nucleotides of the loop of helix 31 of the 16S rRNA 968-970 protected by the enzyme binding to the $30 \mathrm{~S}$ subunit were also protected by the S19 protein (Powers and Noller 1995). The latter is essential for RsmD binding to the 30S subunit (Weitzmann et al. 1991).

\section{Kinetic properties of RsmD}

To measure kinetic characteristics of RsmD methyltransferase, we used multiple turnover reaction conditions. RsmD protein was taken in a catalytic amount, while unmethylated 30 S subunits were taken in excess at varying concentrations. The reaction was started by addition of a RsmD and $\left[{ }^{3} \mathrm{H}\right] \mathrm{SAM}$ solution to the $30 \mathrm{~S}$ subunits. The reaction was stopped by the addition of RNA extraction buffer containing guanidine 


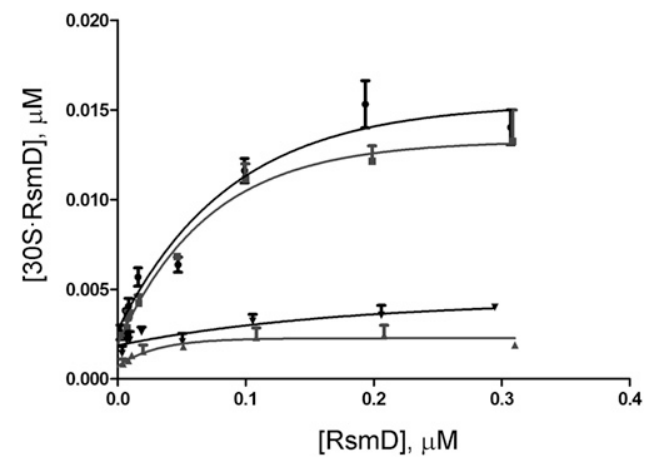

FIGURE 2. Titration curves of $30 \mathrm{~S}$ subunits devoid of G966 methylation with the increasing concentration of RsmD. Unmethylated $30 \mathrm{~S}$ subunits in the presence of sinefungin (squares), unmethylated $30 \mathrm{~S}$ subunits in the presence of S-adenosyl-L-homocystein (SAH) (circles). Methylated 30S subunits in the presence of sinefungin and SAH (triangles pointing up and down, respectively).

chloride at various time points, starting from $30 \mathrm{sec}$ to 24 min. Methylated 16S RNA was extracted, and the amount of radioactive $\left[{ }^{3} \mathrm{H}\right]$ methyl groups included in the 16S RNA was measured by scintillation counting. The obtained data were used to calculate initial reaction rates. LineweaverBurk linearization allowed to calculate kinetic constants (Fig. 4A). It was found that the Michaelis constant of RsmD is $3.3 \pm 0.6 \mathrm{nM}$, while kcat is $0.028 \pm 0.004 \mathrm{~min}^{-1}$.

\section{Thermodynamic characteristics of RsmD binding to SAM, SAH, and sinefungin}

RsmD interacts with the methyl group donor, SAM, and after methyl group transfer to the 16S rRNA, SAH left in the active center of RsmD should exchange for another molecule of SAM. The SAM binding center of methyltransferase could be occupied with the competitive inhibitor, sinefungin, which could not serve as a source of a methyl group. To determine dissociation constants and thermodynamic characteristics of RsmD interaction with these small ligands, we applied isothermal titration calorimetry (ITC).

Ligand-free RsmD solution was titrated with SAM, SAH, or sinefungin solutions in the same buffer (Fig. 5A). SAM binding was shown to be tightest, with $K_{d}=0.58 \mu \mathrm{M}$, while SAH binds four times less tightly, with $K_{d}=2.5 \mu \mathrm{M}$. Abundance of SAM in E. coli was estimated to be $400 \mu \mathrm{M}$, while that of SAH was $1.3 \mu \mathrm{M}$ (Halliday et al. 2010). Such a difference in binding and metabolite abundance ensures a rapid exchange of SAH to SAM following 30 S methylation. The affinity toward sinefungin was shown to be half that of SAH and 8 times less than that of SAM (Fig. 5A).

Binding of SAM is driven by both an enthalpy decrease and entropy increase (Table 1), indicating a significant contribution of all types of interactions, including hydrophobic, possibly provided by both adenine and methyl groups. Removal of the methyl group significantly affects the entropy change, making it negative, which could in- dicate the significance of the methyl group in hydrophobic interactions. The unfavorability of the binding change in $\Delta \mathrm{S}$ was partially compensated by the gain in $-\Delta \mathrm{H}$ (Table 1). Sinefungin binding appeared to be even more enthalpydriven, with a large decrease in entropy (Table 1).

\section{Mutational analysis of the RsmD methyltransferase}

RsmD belongs to the family of Rossmann fold N-methyltransferases which have a conserved arrangement of active site residues (Sergiev et al. 2007). Two faces of the SAM binding cavity were formed by ${ }^{127} \mathrm{DPPF}^{130}$ and ${ }^{58} \mathrm{DxFxGxG}{ }^{64}$ motifs. We introduced mutations into conserved positions ${ }^{58} \mathrm{D},{ }^{128} \mathrm{P},{ }^{129} \mathrm{P}$, and ${ }^{130} \mathrm{~F}$. The following mutations were made: F130S, PP128-129AA, D58A, PPF128-130AAS, and D58A/PP128-129AA. Plasmids encoding mutant proteins were checked to complement knockout of the $r s m D$ gene in vivo (Fig. 5B). Methylation of G966 of the $16 \mathrm{~S}$ rRNA was monitored by reverse transcription of total RNA, extracted from the corresponding strains. Surprisingly, the majority of active site mutants retained the ability to form the $\mathrm{m}^{2} \mathrm{G} 966$ residue. The triple PPF128-130AAS mutation alone was found to abolish methylation.

Catalytic properties of RsmD mutants were tested in vitro in a multiple turnover assay (Fig. 4B-D) similar to that of the wild-type RsmD (Fig. 4A). The results of the experiment are presented in Table 1. Substitution of the conserved proline residues PP128-129AA present in all rRNA guanine-(N2)-methyltransferases (Sergiev et al. 2007), surprisingly, had only a marginal effect on the catalytic ability of the enzyme (Fig. 4C; Table 1). Mutation of D58A, both separately (Fig. 4B; Table 1) and in combination with PP128-129AA (Fig. 4D; Table 1), increased $K_{m}$ toward the 30 S subunit by two orders of magnitude with only a marginal effect on kcat. Measurement of enzymatic

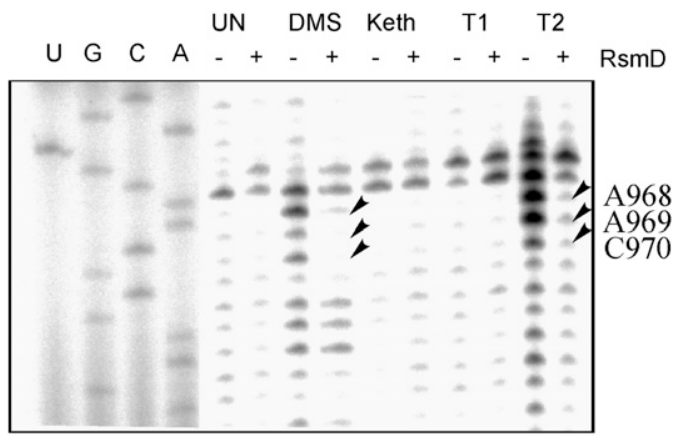

FIGURE 3. Interaction of 30 S ribosomes devoid of G966 methylation with the methyltransferase RsmD in the presence of sinefungin monitored by chemical and enzymatic footprinting. Lanes $A, C, G$, $U$ correspond to sequencing lanes in the presence of corresponding ddNTP; UN corresponds to untreated 30 S subunits of $30 \mathrm{~S} \cdot \mathrm{RsmD}$ complex; DMS, Keth, T1, and T2 each correspond to treatment with dimethyl sulfate, kethoxal, and RNases T1 and T2, respectively; +/indicates the preincubation of unmethylated at G966 30S subunits with RsmD and sinefungin. Protected bases are marked by arrows. 
A.
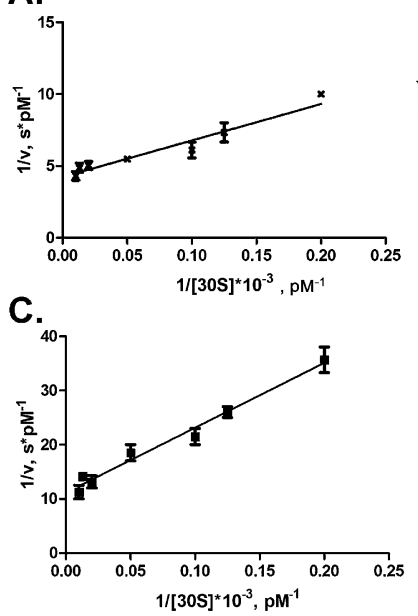

B.
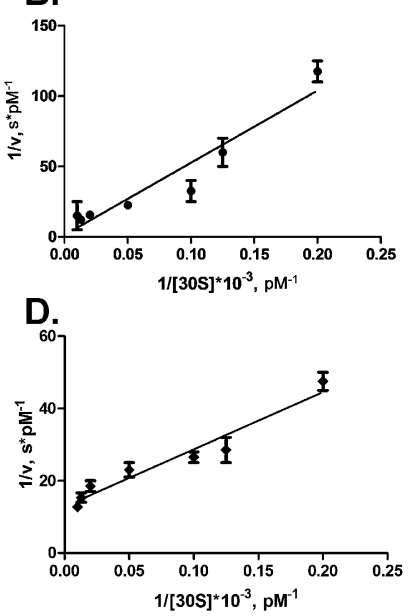

FIGURE 4. Lineweaver-Burk linearization of the Michaelis-Menten kinetics of the 30 S methylation by $(A) \mathrm{RsmD},(B) \mathrm{D} 58 \mathrm{~A},(C)$ PP128129AA, (D) D58A/ PP128-129AA.

properties of the F130S mutant in vitro was impossible due to its instability in solution.

The ability of mutant forms of RsmD to bind the SAM cofactor was tested by ITC (Fig. 5A). Mutations D58A and PP128-129AA decreased the enthalpic contribution to SAM binding, paralleled by an increase in the entropic contribution, which might indicate an increase in flexibility of SAM in its binding pocket. SAM binding to the enzyme with the combination of mutations D58A/PP128-129AA was impossible to measure due to a very small enthalpy of binding.

\section{DISCUSSION}

Ribosomal RNA methyltransferase RsmD operates on the late stage of the $30 \mathrm{~S}$ subunit assembly (Weitzmann et al. 1991) and could modify a completely assembled 30S subunit (Lesnyak et al. 2007). The modification site of RsmD, nucleotide $\mathrm{m}^{2} \mathrm{G} 966$ of the $16 \mathrm{~S}$ rRNA, is located in a deep cleft of the ribosomal decoding center (Berk et al. 2006). Modified $\mathrm{m}^{2} \mathrm{G} 966$ makes contact with the anticodon of the P-site bound tRNA (Moazed and Noller 1986). In the course of translation, the P-site is constantly occupied by tRNA, and it is hard to imagine how RsmD could modify G966 after the $30 \mathrm{~S}$ subunit starts a round of translation. On the other hand, since G966 modification gives an advantage for 30S functionality (D Burakovsky, I Prokhorova, O Sergeeva, P Sergiev, P Milon, A Bogdanov, O Dontsova, and M Rodnina, in prep.), but the unmodified 30S subunit, nevertheless, could be involved in translation (Lesnyak et al. 2007), it is necessary to accomplish modification of the nascent small subunit before its involvement in translation initiation. After completion of modification, it is preferable to avoid RsmD interference with 30 S subunit functioning.

In this work, we investigated functional properties of RsmD and found it to efficiently bind $30 \mathrm{~S}$ subunits unmethylated at
G966 nucleotide. Dissociation constants for RsmD complexes with unmethylated 30 S subunits were found to be in the $30-40 \mathrm{nM}$ range. This showed that binding is approximately two orders of magnitude better than those demonstrated for comparable rRNA methyltransferases RsmC (Sunita et al. 2007), Sgm (Husain et al. 2010), RlmI (Sunita et al. 2008), and ErmC (Maravic et al. 2003). The Michaelis constant of RsmD toward the $30 \mathrm{~S}$ subunit is much better than that of comparable rRNA methyltransferases, while kcat is comparable to other enzymes of this type. RrmJ, which is responsible for methylation of the $2^{\prime}-\mathrm{OH}$ of nucleotide $\mathrm{U} 2552$ of the $23 \mathrm{~S} \mathrm{rRNA}$, displays a $\mathrm{K}_{\mathrm{m}}$ of 0.7 $\mu \mathrm{M}$ relative to the $50 \mathrm{~S}$ subunits and kcat of $0.064 \mathrm{~min}^{-1}$ (Hager et al. 2002). The methyltransferase ErmC, which has a kcat of $0.066 \mathrm{sec}^{-1}$ and $\mathrm{K}_{\mathrm{m}}$ of $2.8 \mu \mathrm{M}$, provides erythromycin resistance (Maravic et al. 2003). RsmD methyltransferase has a kcat of $0.028 \pm 0.004 \mathrm{~min}^{-1}$ and $\mathrm{K}_{\mathrm{m}}$ of $3.3 \pm 0.6 \mathrm{nM}$. Given such tight binding of RsmD to the $30 \mathrm{~S}$ subunits, one might consider the possibility that RsmD binding would interfere with translation initiation. However, we demonstrated how this problem is resolved, since after modification is complete, the $30 \mathrm{~S}$ subunit loses its ability to bind RsmD. 30S subunit involvement in $70 \mathrm{~S}$ ribosome formation makes an additional obstacle for RsmD binding.

Binding of SAM, SAH, and an inhibitor, sinefungin, to RsmD was studied with the help of ITC. Dissociation constants of RsmD with small ligands differed within the borders of one order of magnitude. SAM binding was tightest, about four times better than those of SAH. Given the two

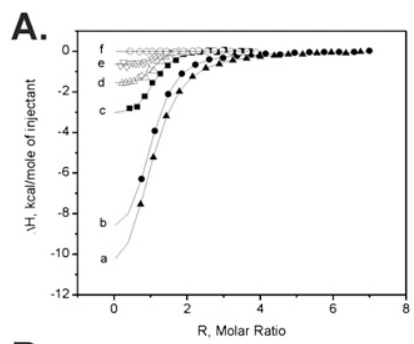

B.

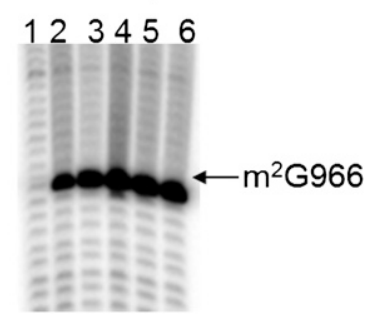

FIGURE 5. (A) ITC of RsmD interaction with small ligands. Shown are ITC profiles for SAM interactions with RsmD (c) and its mutants: PP129-129AA (d), D58A (e), and D58A/PP129-129AA (f), and of RsmD interactions with $\mathrm{Sin}(\mathrm{a})$ and $\mathrm{SAH}$ (b) at $25^{\circ} \mathrm{C}$ in the buffer 20 $\mathrm{mM}$ Hepes $\mathrm{K}$ pH 7.5, $5 \mathrm{mM} \mathrm{Mg}(\mathrm{OAc})_{2}, 200 \mathrm{mM} \mathrm{NH}_{4} \mathrm{OAc}, 10 \%$ glycerol, $5 \mathrm{mM}$ DTT. (B) Reverse transcription analysis of the total rRNA extracted from the knockout RsmD strain transformed with the plasmids coding for the following rsmD mutants: (1) PPF128130AAS, (2) F130S, (3) PP128-129AA, (4) D58A, (5) D58A/PP128129AA, (6) no mutation (control). 
Sergeeva et al.

TABLE 1. Properties of RsmD and its mutant forms

\begin{tabular}{|c|c|c|c|c|c|c|c|}
\hline Protein & Ligand & $\mathrm{N}^{\mathrm{a}}$ & $\mathrm{K}_{\mathrm{d}}, \mu \mathrm{M}^{\mathrm{a}}$ & $\Delta \mathrm{H}, \mathrm{kcal} \cdot \mathrm{M}^{-1}$ & $\Delta \mathrm{S}, \mathrm{cal} \cdot \mathrm{M}^{-1} \mathrm{~K}^{-1}$ & $\mathrm{~K}_{\mathrm{m}}, \mathrm{nM}^{\mathrm{b}}$ & kcat, $\min ^{-1}$ \\
\hline RsmD & SAM & 1,0 & 0,58 & $-3,2$ & 17,8 & $3.3 \pm 0.6$ & $0.028 \pm 0.004$ \\
\hline PP128-129AA & SAM & 1,1 & 0,55 & $-1,6$ & 23,3 & $5.0 \pm 1.1$ & $0.016 \pm 0.008$ \\
\hline D58A & SAM & 1,0 & 0,37 & $-0,7$ & 27,2 & $230 \pm 60$ & $0.055 \pm 0.007$ \\
\hline D58A/PP128-129AA & SAM & & & No signal & & $110 \pm 40$ & $0.038 \pm 0.01$ \\
\hline RsmD & $\mathrm{SAH}$ & 0,9 & 2,50 & $-9,9$ & $-7,4$ & & No data \\
\hline RsmD & $\operatorname{Sin}$ & 1,0 & 5,00 & $-12,5$ & $-17,6$ & & \\
\hline
\end{tabular}

${ }^{a}$ Standard deviations of binding stoichiometry $(\mathrm{N})$ and enthalpy $(\Delta \mathrm{H})$ measurements do not exceed $10 \%$, and those of the affinity constant $\left(\mathrm{K}_{\mathrm{d}}\right)$ measurements do not exceed $20 \%$.

${ }^{\mathrm{b}}$ Related to unmethylated $30 \mathrm{~S}$ subunits.

orders of magnitude prevalence of SAM in the cell, one can imagine that RsmD is predominantly complexed with SAM in vivo, with the rapid exchange of SAH to SAM after modification of the $30 \mathrm{~S}$ subunit. The dissociation constant for SAM binding to RsmD, $0.58 \mu \mathrm{M}$, is comparable to or slightly better than that of other rRNA methyltransferases. The Sgm complex with SAM has a $K_{d}$ of $18 \mu M$ (Husain et al. 2010), the $\mathrm{K}_{\mathrm{d}}$ of the RsmC complex with SAM is $5 \mu \mathrm{M}$ (Sunita et al. 2007 ), and the $K_{d}$ of the RlmI complex with SAM is $3 \mu M$ (Sunita et al. 2008). This difference might not be very essential for functioning of methyltransferases in vitro, since the intracellular concentration of SAM-400 $\mu \mathrm{M}$ (Halliday et al. 2010) - is much greater than the $K_{d}$ for all of these enzymes.

In our study, we performed mutagenesis of the RsmD active site (Fig. 6). The binding site for SAM is conserved in Rossmann fold N-methyltransferases (Sergiev et al. 2007). Peptide motifs (D/E)x(G/F)xGxG and (N/D/S)PP(Y/F/W/ $\mathrm{H})$, characteristic for all methyltransferases of this class, could be found in the RsmD primary structure $\left({ }^{58} \mathrm{DxFxGxG}\right.$ and ${ }^{127} \mathrm{DPPF}$ ). In a substantial number of examples described in the literature, even single amino acid substitutions of the conserved active site residues resulted in the complete loss of enzymatic activity (Hager et al. 2002; Maravic et al. 2003). However, conserved proline residues PP128-129 of RsmD could be substituted for alanines without catalytic activity loss. Moreover, the PP128-129AA mutation does not perturb $\mathrm{K}_{\mathrm{m}}$ and kcat to a significant extent. According to ITC experiments, SAM binding to the PP128-129AA mutant of RsmD was not perturbed either. There is a decrease in enthalpic contribution to the binding, while the entropy contribution was increased. Most likely, this reflects an increase in flexibility of SAM in its binding site. Mutation D58A in the conserved SAM binding site does not abolish methyltransferase activity in vivo and has almost no effect on $K_{d}$ of SAM binding in vitro. This result was unexpected since similar substitution D202A in the RsmC active site abolished SAM binding and reduced catalytic activity by two orders of magnitude (Sunita et al. 2007). Mutation of an equivalent position in DNA methyltransferase EcoRV (Roth and Jeltsch 2001) also inactivated the enzyme. Similar to the PP128-129AA mutation, the D59A mutation increases entropic contribution to binding at the expense of enthalpic and is likely to increase SAM flexibility in its binding site. In contrast to the PP128-129AA mutation, the D58A mutation increased $\mathrm{K}_{\mathrm{m}}$ of RsmD toward $30 \mathrm{~S}$ subunits by two orders of magnitude. It is, thus, likely that the D58 residue helps in 30S subunit binding. Another element essential for the $30 \mathrm{~S}$ subunit binding was found recently in a study on Mycobacterium tuberculosis RsmD homolog Rv2966c (Kumar et al. 2011). This element is the $\beta$-hairpin located on the $\mathrm{N}$ terminus of the enzyme.

Even the RsmD triple D58A/PP128-129AA mutant retains some methyltransferase activity in vivo and in vitro, although it increases $\mathrm{K}_{\mathrm{m}}$ toward the $30 \mathrm{~S}$ subunit by two orders of magnitude and displays insignificant enthalpy of SAM binding. Aromatic amino acid residue following the PP128-129 motif was known to be essential for methyltransferase activity of DNA and RNA methyltransferases, in particular EcoRV (Roth and Jeltsch 2001) and ErmC (Maravic et al. 2003). Only the triple PPF128-130AAS mutation resulted in the complete loss of RsmD methyltransferase activity.

Summarizing the results, we can state that RsmD methyltransferase evolved to possess an ability to bind the $30 \mathrm{~S}$ subunit more tightly than comparable rRNA methyltransferases. This property might be essential for fast binding of unmodified nascent subunits to insure their modification prior to involvement in translation.

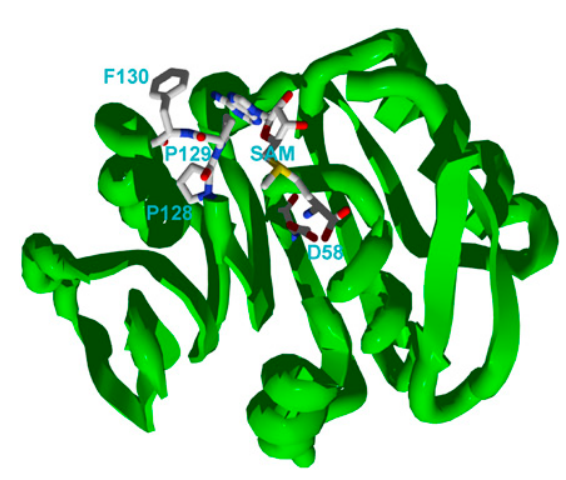

FIGURE 6. Overview of docked SAM inside the substrate binding site of RsmD protein. Shown are SAM molecule and amino acid residues subjected to site-directed mutagenesis in this work. 


\section{MATERIALS AND METHODS}

\section{Strains and plasmids}

The BW25113 parental strain and JW3430 strain, carrying a kanR cassette inserted at the place of the $y h h F$ gene (Baba et al. 2006), later renamed to $\mathrm{rsmD}$, as well as the pCA24yhhF plasmid (Kitagawa et al. 2005) were kindly provided by Dr. H. Mori.

\section{Protein RsmD preparation}

Recombinant N-terminal hexahistidine-tagged RsmD protein was prepared from AG1 E. coli cells, harboring the pCA24N plasmid with the $y h h F$ gene cloned under control of the T5lac promoter (Kitagawa et al. 2005). Cells were grown in LB media at $37^{\circ} \mathrm{C}$ until $\mathrm{A}_{600} 0.5$ and induced by IPTG, $0.5 \mathrm{mM}$ f.c. After induction, cells were grown for an additional $2 \mathrm{~h}$ and lysed by sonication in a buffer of $20 \mathrm{mM}$ Hepes $\mathrm{K} \mathrm{pH} \mathrm{7.5,} \mathrm{0.1 \%} \mathrm{Triton} \times 100,200 \mathrm{mM}$ $\mathrm{NH}_{4} \mathrm{OAc}, 10 \mathrm{mM}$ imidazole. Protein purification on Ni-NTA agarose (Qiagen) was done in the same buffer, containing $10 \mathrm{mM}$ imidazole. After being washed three times with the same buffer containing $40 \mathrm{mM}$ imidazole, the protein was eluted by increasing the imidazole concentration up to $500 \mathrm{mM}$. Eluted RsmD protein was dialyzed in the buffer containing $20 \mathrm{mM}$ Hepes $\mathrm{K}$ pH 7.5, $0.1 \%$ Triton $\times 100,200 \mathrm{mM} \mathrm{NH}_{4} \mathrm{OAc}, 1 \mathrm{mM} \mathrm{Mg}(\mathrm{OAc})_{2}, 5 \mathrm{mM}$ $\beta$-mercaptoethanol, $10 \%$ glycerol. The dialysis was repeated three times to get rid of all SAM, copurified with RsmD. Protein purity was proven by SDS-PAGE. Mutant variants of the RsmD protein were prepared similarly.

\section{S subunits preparation}

The wild-type and JW3430 strain were grown to $\mathrm{A}_{600} 0.6$ in LB media at $37^{\circ} \mathrm{C}$. Cells were harvested by centrifugation and lysed by grinding with $2 \times$ cell mass of aluminium oxide at $4^{\circ} \mathrm{C}$ in the cellopening buffer, $20 \mathrm{mM}$ Hepes $\mathrm{K} \mathrm{pH} \mathrm{7.5,} 100 \mathrm{mM} \mathrm{NH}_{4} \mathrm{Cl}, 10.5$ $\mathrm{mM} \mathrm{Mg}(\mathrm{OAc})_{2}, 3 \mathrm{mM} \beta$-mercaptoethanol. Cell debris and aluminium oxide were pelleted by 30 -min centrifugation at 16,000 $\mathrm{rpm}$ in a JA20 rotor. Lysates were subjected to a 16-h ultracentrifugation in a Ti50 rotor at 33,000 rpm to pellet ribosomes. Ribosomes were resuspended in $20 \mathrm{mM}$ Hepes $\mathrm{K}$ pH 7.5, $10.5 \mathrm{mM}$ $\mathrm{Mg}(\mathrm{OAc})_{2}, 500 \mathrm{mM} \mathrm{NH} \mathrm{m}_{4} \mathrm{OAc}, 7 \mathrm{mM} \beta$-mercaptoethanol buffer. Additionally, ribosomes were pelleted through the $30 \%$ sucrose cushion with the help of ultracentrifugation in Ti50 at 28,000 rpm for $13 \mathrm{~h}$. $70 \mathrm{~S}$ ribosomes were prepared by sucrose gradient centrifugation $(10 \%-40 \%)$ in a Ti15 zonal rotor at 28,000 rpm for 19 h. After that, 70S subunits were pelleted in Ti50 at 50,000 rpm for $24 \mathrm{~h}$. $30 \mathrm{~S}$ subunits were isolated by sucrose gradient centrifugation $(10 \%-40 \%)$ in a Ti15 zonal rotor at $28,000 \mathrm{rpm}$ for $19 \mathrm{~h}$ in the same buffer but with $1 \mathrm{mM} \mathrm{Mg}(\mathrm{OAc})_{2}$. After that, $30 \mathrm{~S}$ subunits were pelleted in Ti50 at 50,000 rpm for $24 \mathrm{~h}$ and dissolved in the buffer containing $50 \mathrm{mM}$ Hepes $\mathrm{K}, 70 \mathrm{mM} \mathrm{NH}_{4} \mathrm{Cl}, 30 \mathrm{mM} \mathrm{KCl}$, $7 \mathrm{mM} \mathrm{Mg}(\mathrm{OAc})_{2}$ (HAKM7). For rRNA preparation, we used phenol extraction in buffer with $300 \mathrm{mM}$ NaOAc, $0.5 \%$ SDS, $5 \mathrm{mM}$ EDTA, followed by ethanol precipitation. The activity of $30 \mathrm{~S}$ subunits reassociated with $50 \mathrm{~S}$ subunits was $>95 \%$ in $\mathrm{fMet}^{-\mathrm{RNA}}{ }^{\mathrm{fMet}}$ binding.

\section{Complex formation}

For complex formation between $30 \mathrm{~S}$ subunits and RsmD methyltransferase, we mixed $0.5 \mu \mathrm{M}$ of $30 \mathrm{~S}$ prepared from the strain lacking the $r s m D$ gene with $1 \mu \mathrm{M}$ of $\mathrm{RsmD}$ in the presence of 5 $\mu \mathrm{M}$ sinefungin in the buffer $50 \mathrm{mM}$ Hepes $\mathrm{K}, 70 \mathrm{mM} \mathrm{NH}_{4} \mathrm{Cl}, 30$ $\mathrm{mM} \mathrm{KCl}, 7 \mathrm{mM} \mathrm{Mg}(\mathrm{OAc})_{2}$ (HAKM7). The mixture was incubated $10 \mathrm{~min}$ at $37^{\circ} \mathrm{C}$. Following the formation of the complex, ultracentrifugation in a $10 \%-30 \%$ sucrose gradient in SW41 at $22,000 \mathrm{rpm}$ for $19 \mathrm{~h}$ was used to isolate the complex. The gradient was fractionated with simultaneous monitoring of optical density at $260 \mathrm{~nm}$. Gradient fractions were analyzed by the method of immunoblotting using anti-His ${ }_{6}$ antibodies (Qiagen) for RsmD protein detection, while anti-S4 antibodies, kindly provided by Dr. R. Brimacombe, were used for detection of ribosomal protein S4. Similar conditions were used to compare efficiencies of RsmD complex formation with unmethylated $30 \mathrm{~S}$ subunits and $70 \mathrm{~S}$ ribosomes, with the sole difference in ultracentrifugation speed being 18,000 rpm.

\section{Calculation of dissociation constant}

For the complex formation, we used $10 \mathrm{nM} 30 \mathrm{~S}$ and 5.3, 9.6, 12, $21,53,110,210$, and $320 \mathrm{nM}$ methyltransferase RsmD concentrations in the presence of $10 \mu \mathrm{M}$ SAH or sinefungin in HAKM7 buffer. Mixtures were incubated $40 \mathrm{~min}$ at $37^{\circ} \mathrm{C}$, and then the complexes were isolated by ultracentrifugation in MLA- 80 rotor at 50,000 rpm for the period of $18 \mathrm{~h}$. The pellets were dissolved in HAKM7 buffer. After measurement of optical density at $260 \mathrm{~nm}$, aliquots containing equal amount of $30 \mathrm{~S}$ subunits were analyzed by ELISA using anti-His ${ }_{6}$ antibodies (Qiagen). "One site binding" analysis of the program GraphPad Prizm was used for the calculation of the dissociation constant.

\section{Chemical and enzymatic probing}

For chemical and enzymatic probing, dimethyl sulfate (DMS), kethoxal, and ribonucleases T1 and T2 were used. The complex of unmethylated $30 \mathrm{~S}$ subunits $(0.4 \mu \mathrm{M})$ with $\mathrm{RsmD}$ protein $(0.8$ $\mu \mathrm{M})$ was formed in the buffer $20 \mathrm{mM}$ Hepes $\mathrm{K}$ pH 7.5, $1 \mathrm{mM}$ $\mathrm{Mg}(\mathrm{OAc})_{2}, 200 \mathrm{mM} \mathrm{NH} \mathrm{OAc}_{4}$ for $30 \mathrm{~min}$ at $37^{\circ} \mathrm{C}$. Following complex formation, $20 \mathrm{nM}$ of either DMS or kethoxal $20 \%$ solution in ethanol was added, and incubation was continued for $10 \mathrm{~min}$ at $37^{\circ} \mathrm{C}$. For the enzymatic probing, 1 unit of T1 ribonuclease and 0.1 unit of $\mathrm{T} 2$ ribonuclease were used. Reactions were stopped and RNA extracted as previously described (Sergiev et al. 2000). For the reverse transcription, $\left[{ }^{32} \mathrm{P}\right] 5^{\prime}$ end-labeled primer complementary to the 996-1012 nt of 16S rRNA was used.

\section{Multiple turnover methylation of unmethylated 30S subunits by recombinant RsmD}

To determine kcat and $\mathrm{K}_{\mathrm{m}}$, recombinant $\mathrm{RsmD}$ in a $0.5 \mathrm{nM}$ final concentration was rapidly mixed with unmethylated $30 \mathrm{~S}$ subunits at $5 \mathrm{nM}, 8 \mathrm{nM}, 10 \mathrm{nM}, 20 \mathrm{nM}, 50 \mathrm{nM}, 80 \mathrm{nM}$, and $100 \mathrm{nM}$ final concentration in the presence of $1000 \times$ excess of $\left[{ }^{3} \mathrm{H}\right] \mathrm{SAM}$. The control sample was without $30 \mathrm{~S}$ subunits. Reactions were mixed on a 96-well microtiter plate with the help of the Janus Extended workstation (Perkin-Elmer). Samples were incubated for $30 \mathrm{sec}$, $1.5,3,6,12$, and $24 \mathrm{~min}$. Following the incubation, the reactions were stopped by adding the guanidine chloride-containing buffer for rRNA isolation (Qiagen). After rRNA isolation (RNeasy Mini Kit, Qiagen), all fractions were analyzed by scintillation counting. Obtained data were analyzed using the Michaelis-Menten equations and following linearization by Lineweaver-Burk. $\mathrm{K}_{\mathrm{m}}$ and 
kcat of RsmD mutant variants were measured and calculated similarly.

\section{Functional activity of mutant RsmD in vivo}

Mutations F130S, PP128-129AA, D58A, PPF128-130AAS, and D58A/PP128-129AA were introduced into the pCA24yhhF plasmid (Kitagawa et al. 2005) coding for RsmD with the help of the QuickChange kit (Stratagene). To monitor G966 methylation in vivo, total RNA was isolated from the cells devoid of $r s m D$ gene on the chromosome, transformed with the pCA24yhhF plasmids containing specified mutations of the active site residues. For the reverse transcription, $\left[{ }^{32} \mathrm{P}\right] 5^{\prime}$ end-labeled primer complementary to the 996-1012 nt of 16S rRNA was used. Reverse transcription was done as previously described (Lesnyak et al. 2007; Sergiev et al. 2011b).

\section{Isothermal titration calorimetry}

ITC measurements were carried out on an iTC200 instrument (MicroCal) at $25^{\circ} \mathrm{C}$ in the buffer, $20 \mathrm{mM}$ Hepes $\mathrm{K} \mathrm{pH} 7.5,5 \mathrm{mM}$ $\mathrm{Mg}(\mathrm{OAc})_{2}, 200 \mathrm{mM} \mathrm{NH} \mathrm{m}_{4} \mathrm{OAc}, 10 \%$ glycerol, $5 \mathrm{mM}$ DTT. Aliquots of ligand solution $(2 \mathrm{~mL})$ were injected into the $0.2 \mathrm{~mL}$ cell containing the protein solution to achieve a complete binding isotherm. The protein concentration in the cell ranged from 10 to $20 \mu \mathrm{M}$, and the ligand concentration in the syringe ranged from 0.2 to $0.75 \mathrm{mM}$. The heat of dilution was measured by additional injections of the ligand after saturation; the values obtained were subtracted from the heat of the complex formation to obtain the effective heat of binding. We fitted the resulting titration curves using MicroCal Origin software (Fig. 5) and determined the dissociation constants $\left(\mathrm{K}_{\mathrm{d}}\right)$, binding stoichiometry $(\mathrm{N})$, and enthalpy $(\Delta \mathrm{H})$ using a nonlinear regression fitting procedure. Consequently, the entropy change $(\Delta \mathrm{S})$ was calculated according to the standard equation.

\section{ACKNOWLEDGMENTS}

We thank Dr. H. Mori, NIG, Japan, for providing us with the knockout strain JW3430 and pCA24yhhF plasmid, encoding RsmD. We also thank Dr. R. Brimacombe, MPIMG, Germany, for anti-S4 antibodies. This work was supported by grants from the Russian Foundation for Basic Research 10-04-01345-a, 11-04-01314-a, 11-04-01018-a, 11-04-12060-ofi from the Russian Ministry of Science 16.512.11.2108, Federal Agency for Science and Innovations 02.740.11.0706, Molecular and Cellular Biology Program of the Russian Academy of Sciences and Moscow State University Development Program PNR 5.13.

Received February 17, 2012; accepted March 6, 2012.

\section{REFERENCES}

Baba T, Ara T, Hasegawa M, Takai Y, Okumura Y, Baba M, Datsenko KA, Tomita M, Wanner BL, Mori H. 2006. Construction of Escherichia coli K-12 in-frame, single-gene knockout mutants: The Keio collection. Mol Syst Biol 2: 2006.0008. doi: 10.1038/msb4100050.

Berk V, Zhang W, Pai RD, Cate JH. 2006. Structural basis for mRNA and tRNA positioning on the ribosome. Proc Natl Acad Sci 103: $15830-15834$

Connolly K, Rife JP, Culver G. 2008. Mechanistic insight into the ribosome biogenesis functions of the ancient protein KsgA. Mol Microbiol 70: 1062-1075.
Decatur WA, Fournier MJ. 2002. rRNA modifications and ribosome function. Trends Biochem Sci 27: 344-351.

Desai PM, Rife JP. 2006. The adenosine dimethyltransferase KsgA recognizes a specific conformational state of the $30 \mathrm{~S}$ ribosomal subunit. Arch Biochem Biophys 449: 57-63.

Ero R, Peil L, Liiv A, Remme J. 2008. Identification of pseudouridine methyltransferase in Escherichia coli. RNA 14: 2223-2233.

Gu XR, Gustafsson C, Ku J, Yu M, Santi DV. 1999. Identification of the 16S rRNA $\mathrm{m}^{5} \mathrm{C} 967$ methyltransferase from Escherichia coli. Biochemistry 38: 4053-4057.

Hager J, Staker BL, Bugl H, Jakob U. 2002. Active site in RrmJ, a heat shock-induced methyltransferase. J Biol Chem 277: 41978-41986.

Halliday NM, Hardie KR, Williams P, Winzer K, Barrett DA. 2010. Quantitative liquid chromatography-tandem mass spectrometry profiling of activated methyl cycle metabolites involved in LuxSdependent quorum sensing in Escherichia coli. Anal Biochem 403: 20-29.

Husain N, Tkaczuk KL, Tulsidas SR, Kaminska KH, Cubrilo S, Maravic-Vlahovicek G, Bujnicki JM, Sivaraman J. 2010. Structural basis for the methylation of G1405 in 16S rRNA by aminoglycoside resistance methyltransferase Sgm from an antibiotic producer: A diversity of active sites in $\mathrm{m}^{7} \mathrm{G}$ methyltransferases. Nucleic Acids Res 38: 4120-4132.

Kitagawa M, Ara T, Arifuzzaman M, Ioka-Nakamichi T, Inamoto E, Toyonaga H, Mori H. 2005. Complete set of ORF clones of Escherichia coli ASKA library (a complete set of $E$. coli K-12 ORF archive): Unique resources for biological research. DNA Res 12: 291-299.

Kumar A, Saigal K, Malhotra K, Sinha KM, Taneja B. 2011. Structural and functional characterization of Rv2966c protein reveals an RsmD-like methyltransferase from Mycobacterium tuberculosis and the role of its N-terminal domain in target recognition. J Biol Chem 286: 19652-19661.

Lesnyak DV, Sergiev PV, Bogdanov AA, Dontsova OA. 2006. Identification of Escherichia coli $\mathrm{m}^{2} \mathrm{G}$ methyltransferases: I. The $y c b Y$ gene encodes a methyltransferase specific for G2445 of the 23 S rRNA. J Mol Biol 364: 20-25.

Lesnyak DV, Osipiuk J, Skarina T, Sergiev PV, Bogdanov AA, Edwards A, Savchenko A, Joachimiak A, Dontsova OA. 2007. Methyltransferase that modifies guanine 966 of the $16 \mathrm{~S}$ rRNA: Functional identification and tertiary structure. J Biol Chem 282: 5880-5887.

Maravic G, Feder M, Pongor S, Flogel M, Bujnicki JM. 2003. Mutational analysis defines the roles of conserved amino acid residues in the predicted catalytic pocket of the rRNA: ${ }^{6} \mathrm{~A}$ methyltransferase ErmC'. J Mol Biol 332: 99-109.

Moazed D, Noller HF. 1986. Transfer RNA shields specific nucleotides in 16S ribosomal RNA from attack by chemical probes. Cell 47: 985-994.

Powers T, Noller HF. 1995. Hydroxyl radical footprinting of ribosomal proteins on 16S rRNA. RNA 1: 194-209.

Roth M, Jeltsch A. 2001. Changing the target base specificity of the EcoRV DNA methyltransferase by rational de novo protein-design. Nucleic Acids Res 29: 3137-3144.

Sergiev PV, Bogdanov AA, Dahlberg AE, Dontsova O. 2000. Mutations at position A960 of E. coli $23 \mathrm{~S}$ ribosomal RNA influence the structure of $5 \mathrm{~S}$ ribosomal RNA and the peptidyltransferase region of 23 S ribosomal RNA. J Mol Biol 299: 379-389.

Sergiev PV, Lesnyak DV, Bogdanov AA, Dontsova OA. 2006. Identification of Escherichia coli $\mathrm{m}^{2} \mathrm{G}$ methyltransferases: II. The $y g j O$ gene encodes a methyltransferase specific for G1835 of the 23 S rRNA. J Mol Biol 364: 26-31.

Sergiev PV, Bogdanov AA, Dontsova OA. 2007. Ribosomal RNA guanine-(N2)-methyltransferases and their targets. Nucleic Acids Res 35: 2295-2301.

Sergiev PV, Serebryakova MV, Bogdanov AA, Dontsova OA. 2008. The $y b i N$ gene of Escherichia coli encodes adenine- $\mathrm{N}^{6}$ methyltransferase specific for modification of A1618 of $23 \mathrm{~S}$ ribosomal RNA, a methylated residue located close to the ribosomal exit tunnel. J Mol Biol 375: 291-300.

Sergiev P, Golovina A, Prokhorova I, Sergeeva O, Osterman I, Nesterchuk M, Burakovsky D, Bogdanov A, Dontsova O. 2011a. 
Modifications of ribosomal RNA: From enzymes to function. In Ribosomes Structure, Function, and Dynamics (ed. M Rodnina et al.), pp. 97-110. Springer, New York.

Sergiev PV, Osterman IA, Prokhorova IV, Nesterchuk MV, Sergeeva OV, Golovina A, Demina IA, Galiamina MA, Serebriakova MV, Dontsova OA. 2011b. Systems biology approach to the functional role of enzymatic modification of bacterial ribosome. Bioorg Khim 37: 81-90.

Sunita S, Purta E, Durawa M, Tkaczuk KL, Swaathi J, Bujnicki JM, Sivaraman J. 2007. Functional specialization of domains tandemly duplicated within $16 \mathrm{~S}$ rRNA methyltransferase RsmC. Nucleic Acids Res 35: 4264-4274.

Sunita S, Tkaczuk KL, Purta E, Kasprzak JM, Douthwaite S, Bujnicki JM, Sivaraman J. 2008. Crystal structure of the Escherichia coli $23 \mathrm{~S}$ rRNA: ${ }^{5} \mathrm{C}$ methyltransferase $\mathrm{RlmI}(\mathrm{YccW}$ ) reveals evolutionary links between RNA modification enzymes. J Mol Biol 383: 652-666.

Tscherne JS, Nurse K, Popienick P, Michel H, Sochacki M, Ofengand J. 1999. Purification, cloning, and characterization of the 16S RNA $\mathrm{m}^{5} \mathrm{C} 967$ methyltransferase from Escherichia coli. Biochemistry 38: 1884-1892.
Urlaub H, Thiede B, Muller EC, Brimacombe R, Wittmann-Liebold B. 1997. Identification and sequence analysis of contact sites between ribosomal proteins and rRNA in Escherichia coli $30 \mathrm{~S}$ subunits by a new approach using matrix-assisted laser desorption/ionizationmass spectrometry combined with $\mathrm{N}$-terminal microsequencing. $J$ Biol Chem 272: 14547-14555.

Weitzmann C, Tumminia SJ, Boublik M, Ofengand J. 1991. A paradigm for local conformational control of function in the ribosome: Binding of ribosomal protein S19 to Escherichia coli $16 \mathrm{~S}$ rRNA in the presence of $\mathrm{S} 7$ is required for methylation of $\mathrm{m}^{2} \mathrm{G} 966$ and blocks methylation of $\mathrm{m}^{5} \mathrm{C} 967$ by their respective methyltransferases. Nucleic Acids Res 19: 7089-7095.

Wrzesinski J, Bakin A, Nurse K, Lane BG, Ofengand J. 1995. Purification, cloning, and properties of the 16S RNA pseudouridine 516 synthase from Escherichia coli. Biochemistry 34: 89048913.

Xu Z, O'Farrell HC, Rife JP, Culver GM. 2008. A conserved rRNA methyltransferase regulates ribosome biogenesis. Nat Struct Mol Biol 15: 534-536. 

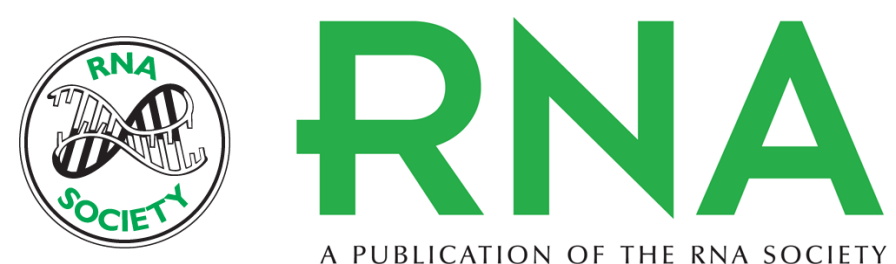

A PUBLICATION OF THE RNA SOCIETY

\title{
Properties of small rRNA methyltransferase RsmD: Mutational and kinetic study
}

Olga V. Sergeeva, Irina V. Prokhorova, Yerdos Ordabaev, et al.

RNA 2012 18: 1178-1185 originally published online April 25, 2012

Access the most recent version at doi:10.1261/rna.032763.112

\author{
References This article cites 30 articles, 8 of which can be accessed free at: \\ http://rnajournal.cshlp.org/content/18/6/1178.full.htmI\#ref-list-1 \\ License
}

Email Alerting Receive free email alerts when new articles cite this article - sign up in the box at the Service top right corner of the article or click here. 